\section{References}

1. Applegate WV. Abdominal cutaneous nerve entrapment syndrome (ACNES): A commonly overlooked cause of abdominal pain. Perman J 2002; 6:20-27.

2. Kanakarajan S, High K, Naaraja R. Chronic abdominal wall pain and ultrasoundguided abdominal cutaneous nerve infiltration: A case series. Pain Med 2011; 12:382-386.

3. Boelens $O B$, Scheltinga MR, Houterman $S$, Roumen RM. Management of anterior cutaneous nerve entrapment syndrome 6 . in a cohort of 139 patients. Ann Surg 2011; 254:1054-1057.

4. Loos MJ, Scheltinga MR, Mulders LG, Roumen RM. The Pfannestiel incision as a source of chronic pain. Obstet Gynecol 7 . 2008; 111:839-845.

5. Nikolajsen L, Sorensen HC, Jensen TS, Kehlet $\mathrm{H}$. Chronic pain following Caesarean section. Acta Anaesthesiol Scand 2004; 48:111-116.
Ducie I, Moxley M, Al-Atar A. Algorithm for treatment of postoperative incisional groin pain after Cesarean delivery or hysterectomy. Obstet Gynecol 2006; 108:2731.

Luijendijk RW, Jeekel J, Storm RK, Schutte PJ, Hop WC, Drogendijk AC, Huikeshoven FJ. The low transverse Pfannenstiel incision and the prevalence of incisional hernia and nerve entrapment. Ann Surg 1997; 225:365-369.

\title{
Q 2. Paresthesia Coverage for Comparing the Inhibition of Somatosensory Evoked Potentials by Spinal Cord Stimulation and Transcutaneous Electrical Nerve Stimulation
}

\section{TO THE EDITOR:}

I read with great interest the article by Wolter et al (1) and appreciated the attempt to answer some important questions on the possible role played by the inhibition of somatosensory evoked potentials (SEPs) by spinal cord stimulation (SCS) used for chronic pain. Although I share with the authors most of their interpretations and opinions, it seems to me that the protocol used to answer the question about the comparison between SCS and transcutaneous electrical nerve stimulation (TENS), was not completely adequate because the 2 stimulations were not applied with the same characteristics. In fact, when applying SCS and TENS, they did not search the complete coverage of paresthesia in the tibial nerve territory, the nerve used for SEPs recordings. Since the coverage of the induced paresthesia is an important prerequisite for SCS efficacy (2), targeting the applied stimulations is indeed very important to adequately compare SCS and TENS. If SCS only covered the tibial nerve territory, the inhibition would be obviously stronger during SCS. In this regard, while it is probable that in the study of Wolter et al (1) SCS effectively covered the whole territory of the tibial nerve, it was unlikely that TENS had the same effect because its electrodes were placed in the medial side of the foot (innervated by the medial plantar nerve, only one of the 2 main terminal branches of the tibial nerve) and at the medial lower leg, $15 \mathrm{~cm}$ above the ankle (in the territory of the saphenous nerve).

The comparison of the inhibitory effects of SCS and TENS on SEPs remains an important point to reach for a better understanding of the differences between the 2 types of electroanalgesia and for a possible use of TENS as a screening tool for SCS (3). Interestingly, it is worth noting that the pathophysiological role played by the large diameter fibers (those investigated by SEPs and activated by both SCS and TENS) in neuropathic pain conditions has gained new importance as a consequence of the official redefinition of neuropathic pain as "pain arising as a direct consequence of a lesion or disease affecting the somatosensory system" (4). This system indeed comprises both the spino-thalamic tract (sensory small fibers) and lemniscal tract (sensory large fibers). It follows that, according to the new definition, a lesion or disease involving the large diameter fibers can be considered, logically speaking, a possible cause of neuropathic pain.

Further studies are then warranted to better compare the inhibitory effect of SCS on SEPs, possibly using 
direct stimulation of the tibial nerve trunk, proximally to the site used for SEPs stimulation, as already used in the past to prove the inhibitory effect of TENS on SEPs (5).

Michelangelo Buonocore, MD

Unit of Clinical Neurophysiology

and Neurodiagnostic Skin Biopsy

\author{
"Salvatore Maugeri" Foundation \\ Scientific Institute of Pavia \\ Via Maugeri 10 \\ 27100 Pavia \\ Italy \\ E-mail: michelangelo.buonocore@fsm.it
}

\section{References}

1. Wolter T, Kieselbach K, Sircar R, Gierthmuehlen M. Spinal cord stimulation inhibits cortical somatosensory evoked potentials significantly stronger than transcutaneous electrical nerve stimulation. Pain Physician 2013; 16:405-414.

2. Holsheimer J, Khan YN, Raza SS, Khan EA. Effects of electrode positioning on perception threshold and paresthesia coverage in spinal cord stimulation. Neuromodulation 2007; 10:34-41.
3. Mathew L, Winfree C, Miller-Saultz D, 5 . Sonty N. Transcutaneous electrical nerve stimulator trial may be used as a screening tool prior to spinal cord stimulator implantation. Pain 2010; 150:327-331.

4. Treede RD, Jensen TS, Campbell JN, Cruccu G, Dostrovsky JO, Griffin JW, Hansson $\mathrm{P}$, Hughes R, Nurmikko T, Serra J. Neuropathic pain: Redefinition and a grading system for clinical and research purposes. Neurology 2008; 70:1630-1635.
Nardone A, Schieppati M. Influences of transcutaneous electrical stimulation of cutaneous and mixed nerves on subcortical and cortical somatosensory evoked potentials. Electroencephalogr Clin Neurophysiol 1989; 74:24-35.

\section{In response:}

We thank Dr. Buonocore for his comments on our study. Indeed, to gain further insights about the mechanisms of action of SCS, also a better knowledge of the effects of SCS as compared to a peripheral stimulation such as TENS, is warranted. Not only from a theoretical point of view, but also from a clinical perspectiv, it would be interesting to know whether or not, on principle, SCS has advantages over a peripheral stimulation. In some conditions (such as postherpetic neuralgia) both SCS (1) and subcutaneous stimulation (or peripheral nerve field stimulation) (2) are possible. Thus closer information about the expectable magnitude of the effect would facilitate the decision for one or the other technique.

The inhibition of SEP amplitudes by SCS is a known phenomen (3-5), but a correlation to the clinical pain relieving effect of SCS has not yet been shown (5).

The examination of SEP amplitudes nonetheless offers the possibility of an objective technical measure of the effects of both TENS and SCS. However, there are a couple of difficulties, inherent to such a comparison, besides the position of the electrodes. The results therefore must be interpreted with caution. Available TENS and SCS devices have different control modes and it can be difficult, if not impossible, to adjust the systems in an equal manner regarding frequency and impulse duration. We noted that point when discussing our results.

Regarding the paresthesia coverage, in our study, the TENS electrodes were fixed at the medial (plantar) side of the foot and approximately $15 \mathrm{~cm}$ above the ankle, so that at least the distal electrode was situated within the tibial nerve territory. The electrode positions were chosen in order to have at least partial cutaneous stimulation of the tibial nerve territory, but at the same time exert a direct stimulation of the tibial nerve. In fact patients under TENS reported a tingling sensation within the tibial nerve territory, also beyond the distal electrode, which we can only explain by direct stimulation of the tibial nerve. We therefore believe, that both TENS and SCS were applied with comparable coverages of the tibial nerve territory.

The main point which supports the comparability of the two modalities in our study setting is, that both 
were applied at the same intensity in relation to minimal and maximal stimulation thresholds.

\author{
Mortimer Gierthmuehlen, MD \\ Department of Neurosurgery \\ University Hospital Freiburg \\ Breisacherstr. 64, 79106 \\ Freiburg, Germany
}

Tilman Wolter, MD

Interdisciplinary Pain Center

University Hospital Freiburg

Breisacherstr. 64, 79106 Freiburg

Germany

E-mail: tilman.wolter@uniklinik-freiburg.de

\title{
References
}

1. Harke $H$, Gretenkort P, Ladleif $\mathrm{HU}$, Koester P, Rahman S. Spinal cord stimulation in postherpetic neuralgia and in acute herpes zoster pain. Anesthesia and analgesia 2002; 94:694-700; table of contents.

2. Sator-Katzenschlager $S$, Fiala $K$, Kress HG, Kofler A, Neuhold J, Kloimstein H, Ilias W, Mozes-Balla EM, Pinter M, Loining N, Fuchs W, Heinze G, Likar R. Subcutaneous target stimulation (STS) in chronic noncancer pain: a nationwide retrospective study. Pain Practice 2010; 10:279-286.

3. Polacek H, Kozak J, Vrba I, Vrana J, Stan- 5 cak A. Effects of spinal cord stimulation on the cortical somatosensory evoked potentials in failed back surgery syndrome patients. Clin Neurophysiol 2007; 118:1291-1302.

4. de Andrade DC, Bendib B, Hattou M, Keravel Y, Nguyen JP, Lefaucheur JP.
Neurophysiological assessment of spinal cord stimulation in failed back surgery syndrome. Pain 2010; 150:485-491.

5. Buonocore M, Bodini A, Demartini L, Bonezzi C. Inhibition of somatosensory evoked potentials during spinal cord stimulation and its possible role in the comprehension of antalgic mechanisms of neurostimulation for neuropathic pain. Minerva Anestesiol 2012; 78:297-302. 